\title{
STRONGLY STRICTLY CYCLIC WEIGHTED SHIFTS
}

\author{
JAMES H. HEDLUND
}

\begin{abstract}
An example is given of a unilateral weighted shift on complex Hilbert space which is strictly cyclic but not strongly strictly cyclic. Similar examples of weighted shifts on the sequence spaces $l^{p}$, for $1<p<\infty$, are indicated.
\end{abstract}

1. Introduction. A unilateral weighted shift operator $T$ on complex separable Hilbert space $H$ is an operator that maps each vector in some orthonormal basis $\left\{e_{n}\right\}_{0}^{\infty}$ into a scalar multiple of the next basis vector: $T e_{n}=w_{n} e_{n+1}$ for each $n$. The shift $T$ is said to be strictly cyclic if there exists a vector $x \in H$ such that $A(T) x=H$, where $A(T)$ is the weakly closed algebra generated by $T$. Let $E_{n}$ denote the span in $H$ of the basis vectors $\left\{e_{i}: i \geqslant n\right\}$. If the restricted operators $T \mid E_{n}$ are all strictly cyclic (for $n=0,1,2, \ldots$ ), then $T$ is said to be strongly strictly cyclic. A. L. Shields, in his survey of weighted shifts, asked if every strictly cyclic weighted shift is strongly strictly cyclic [3, Question 14]. This note gives an example which shows that, even under the additional hypothesis that the weight sequence of the operator is monotonically decreasing to 0 , a strictly cyclic operator $T$ may have a restriction $T \mid E_{1}$ which fails to be strictly cyclic.

2. Statement of results. The notation of [3] is followed. Let $T$ denote a unilateral weighted shift on $H$ with a weight sequence $w_{n}$ such that $w_{0} \geqslant w_{1}$ $\geqslant w_{2} \geqslant \cdots>0$. Define $\beta(0)=1$ and $\beta(n)=w_{0} w_{1} \cdots w_{n-1}$ for $n \geqslant 1$, so that $w_{n}=\beta(n+1) / \beta(n)$. Let $\beta(n, j)=(\beta(n) / \beta(j) \beta(n-j))^{2}$, for $0 \leqslant j \leqslant n$, and let $S_{\beta}(n)=\sum_{j=0}^{n} \beta(n, j)$. In order to determine if $T$ is strictly cyclic it suffices, by the following result, to examine the sequence $\left\{S_{\beta}(n)\right\}$.

Theorem [2, Theorem 3.2], [3, Proposition 32]. If $\left\{w_{n}\right\}$ is monotonically decreasing, then $T$ is strictly cyclic if and only if the sequence $\left\{S_{\beta}(n)\right\}$ is bounded.

Corollary. If $\left\{w_{n}\right\}$ is monotonically decreasing to 0 and if $2<\lim \sup S_{\beta}(n)$ $<\infty$, then $T$ is strictly cyclic but $T \mid E_{1}$ is not. Thus $T$ is strictly cyclic but not strongly strictly cyclic.

Proof. It is no loss of generality to assume that $w_{0}=1$ : if $w_{0} \neq 1$, multiply each weight by $\left(w_{0}\right)^{-1}$ and observe that this change does not affect the values of $\beta(n, j)$ or $S_{\beta}(n)$. The operator $T \mid E_{1}$ is itself a weighted shift, with weights $v_{n}=w_{n+1}$. Define $\alpha(n)$ for $\left\{v_{n}\right\}$ as $\beta(n)$ was defined for $\left\{w_{n}\right\}$, so that $\alpha(0)=1$ $=\beta(1)$ and $\alpha(n)=v_{0} v_{1} \cdots v_{n-1}=\beta(n+1)$. Thus

Received by the editors May 29, 1975 and, in revised form, August 20, 1975.

AMS (MOS) subject classifications (1970). Primary 47B99.

○ American Mathematical Society 1976 


$$
\begin{aligned}
\alpha(n, j) & =(\alpha(n) / \alpha(j) \alpha(n-j))^{2} \\
& =(\beta(n+1) / \beta(j+1) \beta(n+1-j))^{2} .
\end{aligned}
$$

Hence

$$
\begin{aligned}
S_{\alpha}(n)= & \sum_{j=0}^{n} \alpha(n, j)=\sum_{k=1}^{n+1}\left(\frac{\beta(n+1)}{\beta(k) \beta(n+2-k)}\right)^{2} \\
& +\left(\frac{\beta(n+1)}{\beta(n+2)}\right)^{2} \sum_{k=1}^{n+1} \beta(n+2, k)=\left(w_{n+1}\right)^{-2}\left[S_{\beta}(n+2)-2\right]
\end{aligned}
$$

since $\beta(n+2,0)=\beta(n+2, n+2)=1$. If $w_{n} \downarrow 0$ and if $2<\lim \sup S_{\beta}(n)$ $<\infty$, it follows that $\left\{S_{\alpha}(n)\right\}$ is unbounded, so that $T \mid E_{1}$ is not strictly cyclic.

3. Construction. To find weights $\left\{w_{n}\right\}$ which satisfy the conditions of the Corollary, proceed as follows. Note first that, for $n \geqslant 2, S_{\beta}(n)=2$ $+\left(w_{n-1}\right)^{2} K_{n}$, where $K_{n}$ is a function of $w_{0}, \ldots, w_{n-2}$ only. It follows that $S_{\beta}(n)$ is an increasing function of $w_{n-1}$. Consequently, if $w_{0}, \ldots, w_{n-2}$ are already chosen, $w_{n-1}$ may then be chosen to make $S_{\beta}(n)$ take on any value larger than 2 . The trick is to achieve large values of $S_{\beta}(n)$ with a sequence $\left\{w_{n}\right\}$ which is monotonically decreasing to 0 .

Take any value $L>2$, and then choose the $w_{n}$ inductively as follows. Let $w_{0}=w_{1}=1$, and suppose that, for $n \geqslant 3$, the weights $w_{0}, \ldots, w_{n-2}$ have been chosen so that $S_{\beta}(n-1) \leqslant L$. Let $w_{n-1}=w_{n-2}$ if this choice will make $S_{\beta}(n) \leqslant L$ (Case 1$)$; otherwise let $w_{n-1}$ be the smaller of $\frac{1}{2} w_{n-2}$ (Case 2a) and that value which would make $S_{\beta}(n)=L$ (Case 2 b). In either case $S_{\beta}(n) \leqslant L$ and $w_{n-1} \leqslant w_{n-2}$.

For integers $n$ of Case 2, $S_{\beta}(n) \geqslant 3 / 2+L / 4>2$. For, if Case 2a occurs, then $2+\left(w_{n-2}\right)^{2} K_{n}>L$. Hence

$$
\begin{aligned}
S_{\beta}(n) & =2+\left(w_{n-2}\right)^{2} K_{n} / 4 \\
& =3 / 2+\left[2+\left(w_{n-2}\right)^{2} K_{n}\right] / 4>3 / 2+L / 4 .
\end{aligned}
$$

In Case $2 \mathrm{~b}, S_{\beta}(n)=L>3 / 2+L / 4$. Thus $\lim \sup S_{\beta}(n)>2$ if Case 2 occurs infinitely often. This must be true, for otherwise the sequence $\left\{w_{n}\right\}$ is eventually constant. An easy computation then shows that $\lim S_{\beta}(n)=+\infty$, contradicting the fact that $S_{\beta}(n) \leqslant L$. Since $w_{n-1} \leqslant \frac{1}{2} w_{n-2}$ for integers $n$ of Case 2 it follows that the sequence $\left\{w_{n}\right\}$ decreases monotonically to 0 .

4. Remarks. The construction above may easily be modified to make $S_{\beta}(n)$ unbounded. This yields an example of a weighted shift whose weights decrease monotonically to 0 and yet which is not strictly cyclic. (A similar example is given by D. A. Herrero [1].)

Weighted shifts may be defined on the sequence spaces $l^{p}$. The Theorem of Kerlin and Lambert stated above holds for operators on $l^{p}$ with $1<p<\infty$ if it is modified so that $\beta(n, j)$ is defined by $\beta(n, j)=(\beta(n) / \beta(j) \beta(n-j))^{q}$, where $q=p /(p-1)$. (Kerlin and Lambert prove the Theorem in this general form.) The construction above is easily modified to fit this situation. Thus a strictly cyclic weighted shift on $l^{p}$, with $1<p<\infty$, need not be strictly cyclic, even though its weight sequence decreases monotonically to 0 . 


\section{REFERENCES}

1. D. A. Herrero, Strictly cyclic weighted shifts (preprint).

2. E. Kerlin and A. L. Lambert, Strictly cyclic shifts on $l_{p}$, Acta Sci. Math. (Szeged) 35 (1973), 87-94. MR 48 \#7000.

3. A. L. Shields, Weighted shift operators and analytic function theory, Math. Surveys, vol. 13, Amer. Math. Soc., Providence, R.I., 1974, pp. 49-128.

Department of Mathematics, Smith College, Northampton, Massachusetts 01060 